\title{
Anosmia of Covid 19 - A Transient Symptom?
}

\author{
Amrapali Keny Pawar ${ }^{1 *}$, Palak Bhatti ${ }^{2}$ and Prashant Kewle ${ }^{3}$ \\ ${ }^{1}$ Senior Consultant and Head of Department, K.B.Bhabha municipal hospital, Bandra, Mumbai, India \\ ${ }^{2}$ Senior Consultant, Gurunanak Hospital and Research centre, Bandra, Mumbai, India \\ ${ }^{3}$ Senior consultant and Head of Department, Centenary municipal hospital, Govandi, Mumbai, India
}

*Corresponding Author: Amrapali Keny Pawar, Senior Consultant and Head of Department, K.B.Bhabha municipal hospital, Bandra, Mumbai, India.

Received: January 06, 2022; Published: January 13, 2022

\begin{abstract}
Aims and objectives: Covid 19 pandemic brought a host of symptoms in the last 2 years. Amongst these anosmia or loss of smell and taste were some of the striking though less common symptoms. Significant rise in Anosmia with or without onset of upper respiratory tract infection prompted a study in which patients with loss of smell were screened over a period of one year to study the nature of this symptom.

Materials and Methiods: A questionnaire had to be filled by patients visiting in our clinics or through online consultations. 53 patients were selected.

Observations: Our observations showed that in $86.8 \%$ of patients, anosmia was the only symptom and $73.6 \%$ required no specific treatment. In 89.3\% anosmia resolved within 3 months.

Conclusion: Hence it is concluded that anosmia can be used as a marker for COVID 19 testing. It appears to be a transient symptom which resolves spontaneously.

Keywords: Anosmia; loss of smell; COVID 19; SARS-CoV-2; Olfactory dysfunction
\end{abstract}

\section{Introduction}

Anosmia is the inability to perceive smell or odor and affects upto $20 \%$ of the adult population [1]. It may be temporary or permanent and acquired or congenital. Amongst the many causes of anosmia, post viral olfactory loss constitutes around $12 \%$ to $15 \%$ of the cases. However, with the advent of COVID 19 pandemic, there was a significant rise in the cases of anosmia and it was particularly observed in those infected with the SARS-CoV-2 virus, so much so that anosmia was declared as one of the diagnostic symptom of SARS-CoV-2 infection [1-3]. Also, it is noted that affected individuals have reported with a sudden loss of smell which is characteristic [2]. In many of the patients who suffered from sudden onset of loss of smell during the infection of COVID19, the anosmia turned out to be an isolated symptom unlike the anosmia caused by other upper respiratory tract infection or the olfactory loss of common cold which often is accompanied by rhinitis or nasal congestion. This also points out to the fact that the mechanism of anosmia caused by SARS-CoV-2 is distinctively different [2].

The aim of our study was to show that anosmia associated with COVID 19 is self- limiting. However the period of recovery may vary from a few days, weeks to even months. 


\section{Materials and Methods}

This article is based on retrospective study by an online questionnaire survey as well as patients screened by us in our clinic during ENT examination during the course of one year. A customized Proforma was prepared for collection of our data which included the age/ sex of the patients affected with COVID 19 in the past one year, confirmed by either RT-PCR, Antigen test or in some cases HRCT chest as an adjunct. Patients' consent to participate in the study was obtained verbally. Patients were questioned about the onset of anosmia, whether it was associated with loss of taste, the duration of their loss of smell and the medications used for management of their SARS-CoV-2 infection. The severity of anosmia could not be checked as all of them were isolated as soon as the diagnosis was established; also evaluation of severity of smell loss using an online questionnaire would have been subjective and hence that was excluded from our study. Our results were based on the data obtained from an online questionnaire.

\section{Inclusion criteria}

All patients were diagnosed with COVID 19 by swab examination or HRCT chest; patients who presented in the OPD in the past year with sudden anosmia with no apparent known cause for the same; patients who were hospitalized in the past one year for COVID 19 infection.

\section{Exclusion criteria}

Children below 16 years; patients with olfactory or gustatory dysfunction before the onset of COVID 19 pandemic; patients with history of rhinosinusitis and nasal polyps and those who had undergone nasal or sinus surgery.

\section{Results}

Anosmia data Out of 53 patients who suffered from anosmia due to COVID 19,64.2\% percent were females (fig.1). The diagnosis was confirmed by the standard RT-PCR test in majority of the patients, in the rest the diagnosis was confirmed by antigen test. Majority of them presented with fever and loss of smell and/or taste. In these patients, we found cases of isolated loss of smell but none of our patients had loss of taste as a symptom alone. Loss of taste was always accompanied by loss of smell, indicating that loss of taste was just a secondary response to the loss of smell (fig.2). Out of all the patients with post COVID anosmia, 67.9\% had a complete loss of smell and the rest of them suffered from decreased sensation of smell orhyposmia. In majority of our patients anosmia was the presenting or one amongst the initial symptoms of COVID 19 (fig.3). Even if it was not the presenting symptom, in majority of them, anosmia started within a week of the presenting symptom. In 86.8\% (fig.4) loss of smell was the only symptom of COVID 19In 73.6\% of the patients, no treatment was given for loss of smell except a multivitamin (fig.5). In 47.2\% of our patients, anosmia recovered within a few days , only a minority around $9.4 \%$ took around 3 months to recover and another $11.3 \%$ did not recover even after 3 months (fig.6) Only around 3.8percent of our patients were given olfactory training to recover, rest $96.2 \%$ recovered on their own without treatment (fig.7).

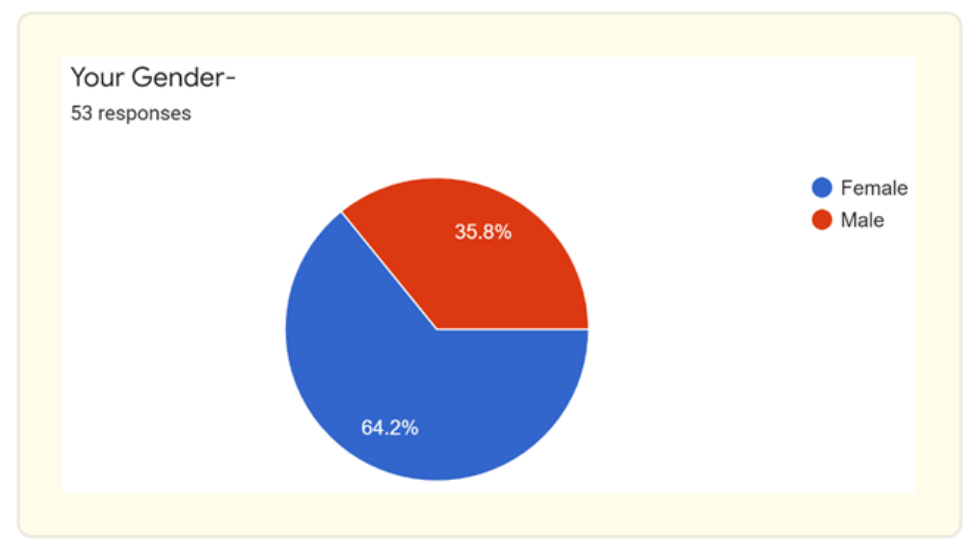


Was the loss of smell accompanied by loss of taste? 53 responses

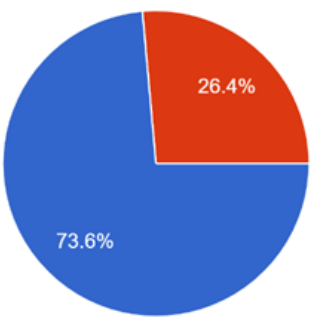

What were the presenting symptom/ symptoms

53 responses

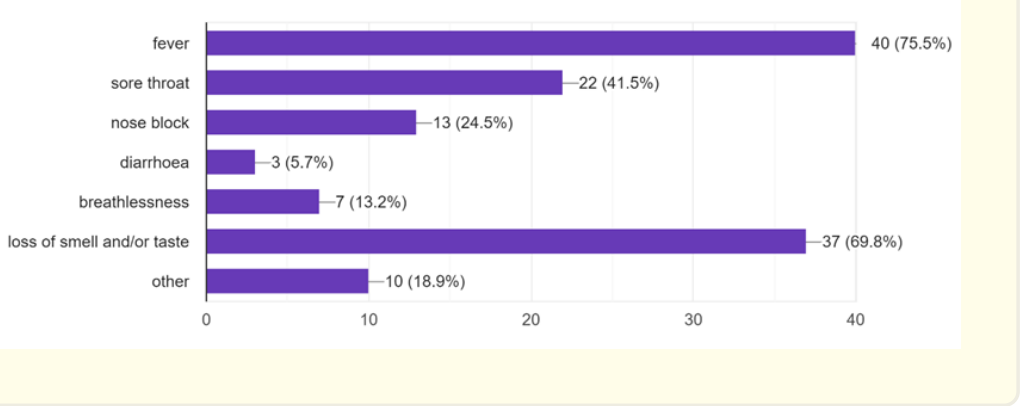

Tick yes if you were not given any treatment for your loss of smell except multivitamin. 53 responses

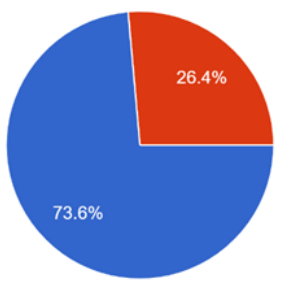

Yes

No 
Was loss of smell the ONLY symptom of COVID 19?

53 responses

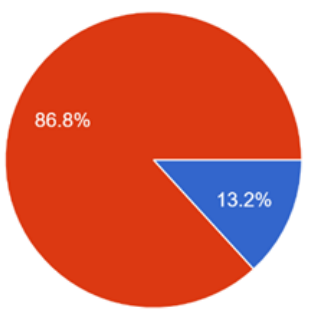

Yes

No

On what day after the onset of anosmia did your smell recover?

53 responses

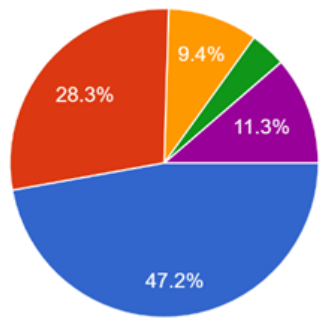

Within a few days

Within 2-3 weeks

Within 3 months

More than 3 months

Did not recover

Were you given olfactory training or smell therapy for your loss of smell?

53 responses

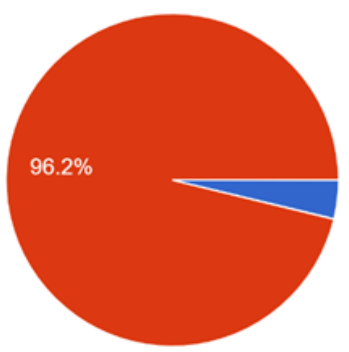

Yes

No

\section{Discussion}

Our study concluded that sudden onset anosmia/hyposmia with no other apparent cause is a potential sign /marker of COVID 19. Its importance lies in the fact that it helps in early detection of the disease and thus may help in implementing early isolation measures which will help in controlling the spread of COVID 19 [4]. 
COVID 19 is infection caused by SARS-CoV-2. The common presenting symptoms of the disease are fever, cough, rhinitis, shortness of breath. By the end of April 2020, loss (or reduction) of the sense of smell (anosmia) was recognized as a marker of COVID 19 infection and was added to the list of COVID 19 symptoms by the WHO [1]. The loss of smell is also accompanied by loss of taste (ageusia).

Anosmia, as a symptom is not paid much attention as it is not life threatening, but a person who suffers from anosmia knows how much it negatively affects the quality of life [1]. Anosmia is often accompanied by loss of sense of taste which in turn prevents the person from enjoying his food leading to malnutrition, weight loss, food poisoning and exposure to dangerous chemicals [2] This in turn affects the overall physical and mental wellbeing of the patient [1].

\section{Pathophysiology of anosmia in COVID 19}

The pathophysiological mechanisms of the cause if anosmia in COVID 19 is still not completely clear but 2 hypothesis have been proposed [4]:

1. Peripheral viral involvement- According to Zou and Collegues [4, 5], the cells of the nasal epithelium have a greater expression of ACE 2 receptors to which SARS-CoV-2 virus is known to bind to infect human beings and hence this results in the olfactory dysfunction reported by a large number of patients affected with COVID 19. The virus binds to these receptors and causes degeneration of the epithelial cells of the nasal mucosa which leads to inflammation and damage of the neural receptors responsible for olfaction.

2. Direct changes in the nervous system by the SARS CoV virus [4, 5]-This hypothesis is the most widely accepted. In an experiment carried out in mice, they were artificially infected with the SARS-CoV-2 virus. It was observed that the virus entered the CNS structures via the Cribriform plate after it attached itself to the nasal epithelium. Thus it reached the olfactory bulb, followed by the olfactory pathway leading to hyposmia or anosmia. As SARS-CoV-2 is structurally similar to SARS CoV virus, it is assumed that it follows the same pathophysiological pathway.

Studies done suggests that the anosmia in COVID 19 is self limiting [7]. Olfactory training may be given in such patients if the recovery does not occur within 2 weeks. However a meta-analysis of 13 studies suggests small to moderate benefit with olfactory training $[7,8]$. The Olfactory Training if incorporated to hasten the recovery of smell may be needed for 8 to 12 weeks [7]. In our study 0T was suggested only to a small number of patients who did not show improvement or recovery from anosmia even after 2 weeks. These patients eventually recovered within 4 to 6 weeks of starting the training. Studies done indicate that SARS-CoV-2 infection can cause olfactory dysfunction but has not known to cause permanent damage of the olfactory function [9]. Our study revealed the same.

There is a noticeable difference between post viral olfactory loss (PVOL) caused by other viruses like Rhinovirus, Coronavirus, Para influenza virus and Ebstein Barr virus in that all these virus cause a gradual loss of smell and/or taste and the OD caused by these viruses may be temporary or sometimes even persistent or permanent [9]. The hallmark of OD caused by SARS-CoV-2 virus is that it is sudden and self limiting (generally resolves within 2-4 weeks). It has been proved by other studies that SARS-CoV-2 infection can produce a transient period of olfactory and gustatory dysfunction but surely does not lead to a permanent loss [9]. Also it has been noticed that a characteristic feature of anosmia caused by SARS-CoV-2 Virus is that it is not associated with rhinorrhea or any other nasal symptom $[10,11]$.

The WHO recommendation is to avoid systemic steroids in severe acute cases of COVID 19 as it suggests possible harm and delayed viral clearance [7].

\section{Conclusion}

Our study concludes that sudden onset anosmia without any apparent cause is a strong and reliable indicator of SARS-CoV-2 infection. It differs from PVOL from other viruses in having the following distinct characteristics: 
1. It is generally the initial symptom.

2. In most of the cases recovery is within a matter of a few days to weeks.

3. It may be the only symptom of SARS-CoV-2 infection.

4. It is transient in nature.

Hence, anosmia can be definitely used as a marker of SARS-CoV-2 infection, although of course, confirmation by the routine diagnostic tests like RT-PCR or antigen testing would be necessary. But it is a good marker for screening patients affected with COVID 19, as also for early diagnosis and isolation of the patient affected with the same. Anosmia caused during the course of COVID 19 infection need not be a cause of worry, since it has generally proven to be self-limiting.

\section{Abbreviations}

OT = Olfactory Training

OD = Olfactory Dysfunction

PVOL = Post Viral olfactory Loss

\section{References}

1. Heike Rebholz., et al. "Loss of Olfactory Function- Early Indicator for Covid -19, Other Viral Infections and Neurodegenerative Disorders". Frontiers in Neurology (2020).

2. Isaias Glezer., et al. "Viral Infection and Smell Loss: The Case of COVID-19”. Journal of Neurochemistry 157.4 (2021): $930-943$.

3. Christina Menni., et al. "Real Time Tracking of Self Reported Symptoms to Predict Potential COVID 19". Nature Medicine 26 (2020): 1037-1040.

4. Paulo Roberto da Silva Junior., et al. "Anosmia and COVID-19: perspectives on its association and the pathophysiological mechanisms involved". The Egyptian Journal of N4eurology, Psychiatry and Neurosurgery 57.1 (2021): 8.

5. Xin Zou., et al. "Single cell RNA -seq data analysis on the receptorACE2 expression reveals the potential risk of different human organs vulnerable to 2019-nCoV infection". Front. Med 14 (2020): 185-92.

6. Netland J., et al. "Severe acute respiratory syndrome coronavirus infection causes neuronal death in the absence of encephalitis in mice transgenic for human ACE2". J Virol 82.15 (2008): 7264-75.

7. Claire Hopkins., et al. "Early Recovery Following New Onset Anosmia During the COVID-19 Pandemic-an Observational Cohort Study". Journal of Otolaryngology-Head \& Neck Surgery 49.26 (2020).

8. Sorokowska A., et al. "Effects of Olfactory Training: a metaanalysis". Rhinology 55.1 (2017): 17-26.

9. Gamba Paolo. “Does COVID-19 Cause Permanent Damage to Olfactory and Gustatory Function?”. Medical Hypothesis 143 (2020): 110086.

10. Mohamed A Amer., et al. "Early Recovery Patterns of Olfactory Disorders in COVID-19 Patients; a Clinical Cohort Study". Am J Otolaryngol 41.6 (2020): 102725.

11. Lechien JR., et al. "Olfactory and Gustatory Dysfunctions as a Clinical Presentations of Mild to Moderate forms of the Coronavirus Disease (COVID-19)”. Eur Arch Otorhinolaryngol 277.8 (2020): 2251-2261.

Volume 2 Issue 2 February 2022

(c) All rights are reserved by Amrapali Keny Pawar., et al. 